\title{
Free Breathing Real-Time Cardiac Cine Imaging With Improved Spatial Resolution at $3 \mathrm{~T}$
}

\author{
Felix Schwab, PhD,* Florian Schwarz, MD, † Olaf Dietrich, PhD, * Titus Lanz, PhD, $\neq$ \\ Frank Resmer, PhD, $\neq$ Tobias Wichmann, MSc, $\neq$ Bernd J. Wintersperger, MD, $\dagger \xi$ Fabian Bamberg, MD, $\dagger \| 1$ \\ Konstantin Nikolaou, MD, † Maximilian Reiser, MD, FACR, FRCR, $\dagger \|$ and Daniel Theisen, MD $\dagger \|$
}

\begin{abstract}
Objectives: The aim of this study was to evaluate free-breathing single-shot real-time cine imaging for functional cardiac imaging at $3 \mathrm{~T}$ with increased spatial resolution. Special emphasis of this study was placed on the influence of parallel imaging techniques.

Materials and Methods: Gradient echo phantom images were acquired with GRAPPA and modified SENSE reconstruction using both integrated and separate reference scans as well as TGRAPPA and TSENSE. In vivo measurements were performed for GRAPPA reconstruction with an integrated and a separate reference scan, as well as TGRAPPA using balanced steady-state free precession protocols. Three clinical protocols, $\mathrm{rtLRInt}\left(T_{\text {res }}=51.3\right.$ milliseconds; voxel, $\left.2.5 \times 5.0 \times 10 \mathrm{~mm}^{3}\right)$, rtMRSep $\left(T_{\text {res }}=48.8\right.$ milliseconds; voxel, $1.9 \times 3.1 \times$ $\left.10 \mathrm{~mm}^{3}\right)$, and rtHRSep $\left(T_{\text {res }}=48.3\right.$ milliseconds; voxel, $\left.1.6 \times 2.6 \times 10 \mathrm{~mm}^{3}\right)$, were investigated on 20 volunteers using GRAPPA reconstruction with internal as well as separate reference scans. End-diastolic volume, end-systolic volume, ejection fraction, peak ejection rate, peak filling rate, and myocardial mass were evaluated for the left ventricle and compared with an electrocardiogram-triggered segmented readout cine protocol used as standard of reference. All studies were performed at $3 \mathrm{~T}$.
\end{abstract}

Results: Phantom and in vivo data demonstrate that the combination of GRAPPA reconstruction with a separate reference scan provides an optimal compromise of image quality as well as spatial and temporal resolution. Functional values ( $P$ values) for the standard of reference, rtLRInt, rtMRSep, and rtHRSep end-diastolic volume were $141 \pm 24 \mathrm{~mL}, 138 \pm 21 \mathrm{~mL}, 138 \pm 19 \mathrm{~mL}$, and $128 \pm 33 \mathrm{~mL}$, respectively $(P=0.7,0.7,0.4)$; end-systolic volume, $55 \pm 15 \mathrm{~mL}, 61 \pm 14 \mathrm{~mL}, 58 \pm 12 \mathrm{~mL}$, and $55 \pm 20 \mathrm{~mL}$, respectively $(P=0.23$, $0.43,0.62)$; ejection fraction, $61 \% \pm 5 \%, 57 \% \pm 5 \%, 58 \% \pm 4 \%$, and $56 \% \pm 8 \%$, respectively $(P=0.01,0.11,0.06)$; peak ejection rate, $481 \pm 73 \mathrm{~mL} / \mathrm{s}, 425 \pm 62 \mathrm{~mL} / \mathrm{s}$, $434 \pm 67 \mathrm{~mL} / \mathrm{s}$, and $381 \pm 86 \mathrm{~mL} / \mathrm{s}$, respectively $(P=0.03,0.04,0.01)$; peak filling rate, $555 \pm 80 \mathrm{~mL} / \mathrm{s}, 480 \pm 70 \mathrm{~mL} / \mathrm{s}, 500 \pm 70 \mathrm{~mL} / \mathrm{s}$, and $438 \pm 108 \mathrm{~mL} / \mathrm{s}$, respectively $(P=0.007,0.05,0.004)$; and myocardial mass, $137 \pm 26 \mathrm{~g}, 141 \pm$ $25 \mathrm{~g}, 141 \pm 23 \mathrm{~g}$, and $130 \pm 31 \mathrm{~g}$, respectively $(P=0.62,0.54,0.99)$.

Conclusions: Using a separate reference scan and high acceleration factors up to $R=6$, single-shot real-time cardiac imaging offers adequate temporal and spatial resolution for accurate assessment of global left ventricular function in free breathing with short examination times.

Received for publication June 11, 2012; and accepted for publication, after revision, October 26, 2012.

From the *Josef Lissner Laboratory for Biomedical Imaging, $†$ Institute for Clinical Radiology, Ludwig-Maximilians-University Hospital Munich, Munich; $¥$ Rapid Biomedical GmbH, Rimpar; $\S$ Department of Medical Imaging, University of Toronto, Toronto Ontario, Canada; and ||Munich Heart Alliance, Munich, Germany.

Conflict of interest and sources of funding: The study was supported by the Bayerische Forschungsstiftung (BFS), by the DZHK (Deutsches Zentrum für Herz-KreislaufForschung-German Centre for Cardiovascular Research), and by the BMBF (German Ministry of Education and Research).

The authors report no conflicts of interest.

Reprints: Felix Schwab, PhD, Josef Lissner Laboratory for Biomedical Imaging, Institute for Clinical Radiology, Ludwig-Maximilians-University Hospital Munich, Marchioninstrasse 15, 81377 Munich, Germany. E-mail: felix.schwab@ med.uni-muenchen.de.

Copyright (C) 2013 by Lippincott Williams \& Wilkins

ISSN: 0020-9996/13/4803-0158
Key Words: cardiac MRI, left ventricular function, parallel imaging, real-time imaging

(Invest Radiol 2013;48: 158-166)

P arameters of global left ventricular (LV) function are essential for the assessment of potential ventricular pathology, permitting assessment of treatment response and potentially allowing prediction of patient outcomes in various cardiac diseases. ${ }^{1,2}$ The precise and reproducible determination of these parameters is therefore of paramount importance in clinical routine. Cardiac cine magnetic resonance imaging (MRI) with steady-state free precession (SSFP) pulse sequences is the standard of reference for noninvasive evaluation of cardiac function..$^{3-5}$ To obtain accurate measures of LV function, a temporal resolution of $T_{\text {Res }}$ of 50 milliseconds or less is recommended, ${ }^{6}$ whereas the in-plane spatial resolution should be better than $3 \times 3 \mathrm{~mm}^{2}$.

The most commonly used procedure is to apply a segmented $\mathrm{k}$-space readout, in which only segments of the entire k-space data set are read out in a single cardiac cycle. Every single image therefore comprises elements of several consecutive cardiac cycles. This approach requires a regular heartbeat and a consistent cardiac position during a breath-hold. For full ventricular coverage, typically multiple consecutive breath-holds are required. In cases of cardiac arrhythmia, limited patient breath-hold capabilities, or limited patient compliance (notably in young children), it therefore appears advantageous to use a single-shot real-time (RT) protocol under free breathing, in which the complete k-space of an image is read out per cardiac phase and heartbeat, and hence, true RT images are acquired. Such protocols have been investigated at $1.5 \mathrm{~T},{ }^{7-11}$ but a thorough analysis at $3 \mathrm{~T}$, where the higher signal-to-noise ratio (SNR) enables the use of higher parallel-imaging acceleration factors, has not yet been performed. On the other hand, studies at $3 \mathrm{~T}$ may be compromised by the appearance of banding artifacts, and the increased energy deposition in the body requires lower flip angles, which prevents from taking full advantage of the improved SNR. Previous studies for segmented readout approaches have investigated the potential gain ${ }^{12-17}$ and concluded that image quality and SNR are significantly improved at $3 \mathrm{~T}$ compared with $1.5 \mathrm{~T}$.

In addition, the analysis of the standard parameters enddiastolic volume (EDV), end-systolic volume (ESV), and ejection fraction (EF) only may not be sufficient for diagnosis of cardiac dysfunction. It is, for example, well known that approximately $50 \%$ of patients presenting with heart failure have a preserved EF. ${ }^{18} \mathrm{~A}$ more thorough diagnosis should include also the assessment of additional parameters reflecting global systolic and diastolic function, such as the peak ejection rate (PER) and the peak filling rate (PFR), which can be calculated from the volume-time curves (VTCs) obtained from complete segmentation of the left ventricle for all cardiac phases.

The purpose of this study was therefore to investigate the potential of RT cardiac MRI for the evaluation of complete global systolic and diastolic cardiac function at $3 \mathrm{~T}$, with special emphasis on the influence of various parallel imaging techniques. 


\section{MATERIALS AND METHODS}

The study consisted of phantom measurements and in vivo experiments in healthy volunteers. All measurements were performed using a clinical 3-T scanner (Magnetom Verio; Siemens Healthcare, Erlangen, Germany) and a dedicated 32-channel cardiac array coil (RAPID Biomedical GmbH, Rimpar, Germany). The coil consisted of an anterior and posterior unit, each containing 16 elements arranged in a 2-dimensional array. The flexible anterior element was geometrically optimized to specifically allow coverage of the heart and measured $19 \times 27 \mathrm{~cm}^{2}$, whereas the solid posterior element measured $32 \times 37 \mathrm{~cm}^{2}$. The study was approved by the local institutional review board and was carried out according to its guidelines. Written informed consent was obtained from each volunteer.

\section{Protocol Implementation and Testing}

Phantom studies were performed on a phantom consisting of 3 bottles of aqueous $\mathrm{NiCl}_{2}$ solution, simulating the chest and arms, in an axial view with a gradient echo sequence to avoid the banding artifacts seen in SSFP protocols (echo time/repetition time, 2.98/5.96 milliseconds; matrix size, $192 \times 192$; field of view, $400 \times 400 \mathrm{~mm}^{2}$; slice thickness, $8 \mathrm{~mm}$; slice gap, $2 \mathrm{~mm}$; bandwidth, $1240 \mathrm{~Hz} /$ pixel; flip angle, $12^{\circ}$; spatial resolution, $2.1 \times 2.1 \times 10 \mathrm{~mm}^{3}$ ). Parallel imaging acceleration factors $(R)$ ranged from 4 to 10 for right-to-left $(\mathrm{R}>>\mathrm{L})$ phase encoding and from 5 to 10 for anterior-to-posterior $(\mathrm{A}>>\mathrm{P})$ phase encoding. Temporal resolution $\left(T_{\text {res }}\right)$ ranged from 267 milliseconds for $R=4$ to 105 milliseconds for $R=10$. To calculate the SNR, 200 images were acquired for both GRAPPA ${ }^{19}$ and modified SENSE (mSENSE ${ }^{20}$ ) reconstruction methods, each combined with both integrated and separate reference scans, as well as TGRAPPA $^{21}$ and TSENSE. ${ }^{22}$ In addition, nonaccelerated images were acquired as a baseline for SNR evaluation.

In addition, 3 RT SSFP protocols were implemented based on the results of the phantom studies to investigate the influence of the reference scan acquisition method in vivo in 5 healthy volunteers:

1. rtMRInt (RT medium resolution with integrated reference scan)

2. rtMRTGRAPPA (RT medium resolution with TGRAPPA)

3. rtMRSep (RT medium resolution with separate reference scan)

All parameters were kept fixed (Table 1), and only the acquisition of the parallel-imaging reference data was varied. Thus, the SNR is expected to be approximately equal and only the impact of reconstruction artifacts can be assessed.

\section{Volumetric Validation in Volunteers}

A total of 20 healthy volunteers were examined under free breathing with 3 optimized RT SSFP sequence protocols based on the results of the first part of the study to demonstrate the possibilities and limitations of this method:

1. rtLRInt (RT low resolution with integrated reference scan): temporal resolution $T_{\mathrm{Res}}$ of 51.3 milliseconds and spatial resolution of $2.5 \times 5.0 \times 10 \mathrm{~mm}^{3}$

2. rtMRSep (RT medium resolution with separate reference scan): temporal resolution $T_{\mathrm{Res}}$ of 48.8 milliseconds and spatial resolution of $1.9 \times 3.1 \times 10 \mathrm{~mm}^{3}$

3. rtHRSep (RT high resolution with separate reference scan): temporal resolution $T_{\text {Res }}$ of 48.3 milliseconds and spatial resolution of $1.6 \times 2.6 \times 10 \mathrm{~mm}^{3}$

Detailed protocol parameters are explicitly listed in Table 1. Each protocol was separately compared with an electrocardiogramtriggered segmented cine protocol (segmInt, with $T_{\text {Res }}=30.4$ milliseconds, spatial resolution of $1.6 \times 1.6 \times 10 \mathrm{~mm}^{3}$ ), serving as standard of reference. The number of slices investigated was adjusted to the size of the heart and varied from 10 to 12 for adequate coverage. The RT protocols were multislice protocols, whereas the segmented cine sequence was a single-slice acquisition, thus requiring 10 to 12 breath-holds. All protocols were repeated to assess reproducibility.

To address off-resonance ("banding") artifacts present when using SSFP at $3 \mathrm{~T}$, which have been well described in the literature, ${ }^{13}$ a segmented readout frequency scout protocol was implemented, which simulated various frequency offsets from -300 to $300 \mathrm{~Hz}$. The optimal frequency shift was chosen visually by the operator.

\section{Data Analysis: Protocol Implementation and Testing}

Because of inhomogeneous noise amplification in parallel imaging, SNR quantification was performed on a pixel-by-pixel basis. Signal-to-noise ratio was analyzed by averaging over all 200 images for the signal and by taking the standard deviation of the signal-time course of each pixel as the noise. In addition to the acceleration factor, the coil and signal properties then depend on 2 quantities, namely, the base $\mathrm{SNR}\left(\mathrm{SNR}_{1}\right)$ for nonaccelerated acquisition and the g-factor. ${ }^{22,23}$ The g-factor is a coil- and setup-specific quantity with values of 1 or greater that describes the effect of noise correlation and coil sensitivities when using parallel imaging. A

TABLE 1. Acquisition Parameters of all Protocols Used in the In Vivo Measurements

\begin{tabular}{|c|c|c|c|c|c|c|}
\hline & $\begin{array}{c}\text { rtMRInt } \\
\text { (Implementation } \\
\text { and Testing) }\end{array}$ & $\begin{array}{c}\text { rtMRTGRAPPA } \\
\text { (Implementation } \\
\text { and Testing) }\end{array}$ & $\begin{array}{c}\text { rtMRSep } \\
\text { (Implementation } \\
\text { and Testing) }\end{array}$ & $\begin{array}{c}\text { rtLRInt } \\
\text { (Validation) }\end{array}$ & $\begin{array}{c}\text { rtHRSep } \\
\text { (Validation) }\end{array}$ & $\begin{array}{c}\text { segmInt } \\
\text { (Validation, Reference) }\end{array}$ \\
\hline TE/TR, ms & $1.37 / 2.74$ & $1.37 / 2.74$ & $1.37 / 2.74$ & $1.29 / 2.56$ & $1.43 / 2.86$ & $1.47 / 2.94$ \\
\hline Matrix & $192 \times 132$ & $192 \times 132$ & $192 \times 132$ & $144 \times 100$ & $224 \times 154$ & $224 \times 168$ \\
\hline Phase resolution, \% & 61 & 61 & 61 & 48 & 58 & 100 \\
\hline Voxel, $\mathrm{mm}^{3}$ & $1.9 \times 3.1 \times 10$ & $1.9 \times 3.1 \times 10$ & $1.9 \times 3.1 \times 10$ & $2.5 \times 5.0 \times 10$ & $1.6 \times 2.6 \times 10$ & $1.6 \times 1.6 \times 10$ \\
\hline View share & No & No & No & Yes & No & No \\
\hline$R$ & 5 & 5 & 5 & 3 & 6 & 2 \\
\hline Reference lines & 30 & - & 60 & 12 & 96 & 24 \\
\hline Reference scan & Internal & TGRAPPA & Separate & Internal & Separate & Internal \\
\hline Bandwidth, Hz/px & 1240 & 1240 & 1240 & 1240 & 1116 & 797 \\
\hline Flip angle & $53^{\circ}$ & $53^{\circ}$ & $53^{\circ}$ & $53^{\circ}$ & $53^{\circ}$ & $50^{\circ}$ \\
\hline
\end{tabular}

TE indicates echo time; TR, repetition time; FOV, field of view. 
theoretical calculation of the g-factor is challenging because necessary inputs are not generally available on the scanner, but based on the SNR analysis, g-factor maps can be calculated ${ }^{24}$ on a pixelby-pixel basis for all acceleration factors $R$ by

$$
g_{R}=\frac{S N R_{1}}{S N R_{R} \sqrt{R}}
$$

where $\mathrm{SNR}_{R}$ is the SNR map at acceleration factor $R$. For a more quantitative assessment of g-factors, profiles were calculated along a line through all 3 phantoms. This was done for images using a separate reference scan and GRAPPA reconstruction only, because this represented the optimal combination. Offline postprocessing was performed on software written in-house using Matlab (The Mathworks, Natick, MA).

In vivo measurements were assessed visually considering image quality and artifacts.

\section{Data Analysis: Volumetric Validation in Volunteers}

The short-axis images of all 20 volunteers were analyzed by 2 experienced readers (F.S., F.S.). Segmentation was performed offline for all protocols using the freely available software Segment version 1.8 R1405 (http://segment.heiberg.se), ${ }^{25}$ and results were averaged over both readers as well as both measurements. Segmentation was performed over the entire cardiac cycle, resulting in a volume-time curve.

In addition to EDV and ESV, which were chosen as the largest and smallest values on this curve, the PFR and the PER were calculated from the maximal positive/negative slopes in diastole and systole, respectively. Finally, LV mass was averaged from the myocardial mass (LVM) measured over all time points.

Image quality was assessed and scored on a 4-point scale (1, excellent quality; 2 , good quality; 3 , moderate artifacts, but diagnostic; 4, heavy artifacts, nondiagnostic).

\section{Statistical Analysis}

Statistical analysis was performed using code written in Matlab as well as in R (www.r-project.org). Correlation between RT protocols and the standard of reference was assessed individually using a Wilcoxon rank sum test, where a $P$ value of less than 0.05 was considered to indicate a statistically significant difference. In addition, Bland-Altman plots were produced to assess systematic and random uncertainties.

Finally, intraobserver and interobserver agreements as well as reproducibility were assessed by calculating intraclass correlation coefficients (ICCs) for all protocols and parameters.

\section{RESULTS}

\section{Protocol Implementation and Testing}

The resulting g-factor maps for different combinations of reconstruction algorithms and reference scans using a rather high acceleration factor of $R=6$ are shown in Figure 1 . In the static phantom, images reconstructed with integrated and separate scan were essentially equal and only one such image is shown. Best results were obtained when using GRAPPA and an internal or separate scan, whereas a higher level of reconstruction noise and artifacts appear with SENSE or TSENSE and particularly with TGRAPPA. Therefore, subsequent SNR analysis focused on these combinations. Results for the g-factor profiles for all applied acceleration factors are given in Figure 2. For $R$ values of 6 or less, g-factors are approximately 1 , whereas for higher acceleration factors such as $R=10$, g-factors increase up to 10 . It is important to note that the dedicated cardiac array has an optimized geometry, which results in lower g-factor values and a higher base SNR and therefore facilitates the use of higher acceleration factors. A comparison of $A>>P$ and $\mathrm{R}>>\mathrm{L}$ phase encoding directions ${ }^{24}$ shows that the former is

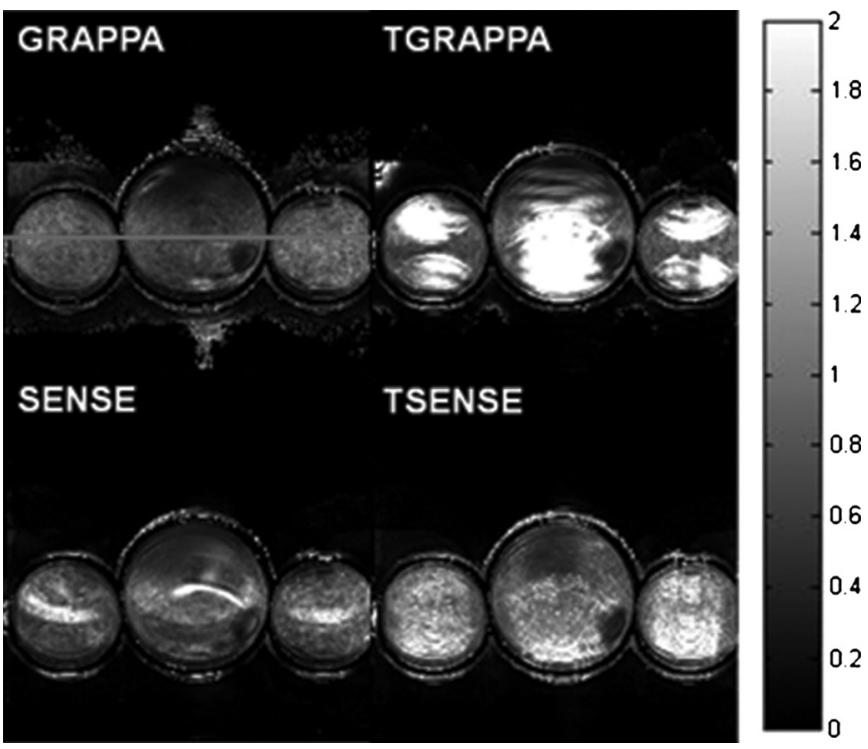

FIGURE 1. Comparison of g-factor maps of a phantom for an acceleration factor of $R=6$. Images correspond to GRAPPA (top) and SENSE (bottom). Internal and separate reference scans do not differ (only results with internal reference scans shown) and show fewer artifacts (left) than TGRAPPA/TSENSE (right). The red line demonstrates the cut along which the profiles presented in Figure 2 were calculated.

preferable for the setup under investigation. Our own analysis confirms these results (Fig. 2), and $\mathrm{A}>>\mathrm{P}$ encoding was therefore applied for the remainder of the study.

The quality of the various reference scans is compared in Figure 3, which demonstrates short-axis images acquired with the protocols rtMRInt, rtMRTGRAPPA, and rtMRSep. The image with the integrated reference scan is almost free of artifacts, whereas minor artifacts are present for the separate reference scan and even stronger ones in the image using TGRAPPA reconstruction.

\section{Volumetric Validation in Volunteers}

Exemplary short-axis images for all protocols rtLRInt, rtMRSep, and rtHRSep as well as segmented readout are shown in Figure 4. The rtLRInt image appears somewhat blurry, and the papillary muscles, in particular, cannot be well delineated. However, the contrast-to-noise ratio between the blood and myocardium appears largest. Mean values over all subjects are summarized in Table 2. Quantitative results generally agreed well between all 3 RT protocols and the standard of reference; statistically significant differences were found only for the PFR and PER of all 3 RT protocols, as well as for the EF determined with the low-resolution protocol.

Bland-Altman plots of all quantitative results are shown in Figures 5 and 6, indicating also parameter variance for all protocols. In general, with the exception of PFR and LVM, the random error increases with the increasing spatial resolution of the protocols. At the same time, except for EDV and PER, the systematic bias decreases. Hence, the preferred RT protocol is rtMRSep, where values for EDV, ESV, EF, and LVM are consistent with the standard of reference, whereas PER and PFR are not. The systematic and random errors shown in the Bland-Altman plots are displayed in Table 3.

Finally, ICCs for all quantitative data are summarized in Table 4. Intraobserver and interobserver reliability and reproducibility are good to excellent for both the standard of reference as well as the preferred medium-resolution protocol rtMRSep when analyzing EDV, 

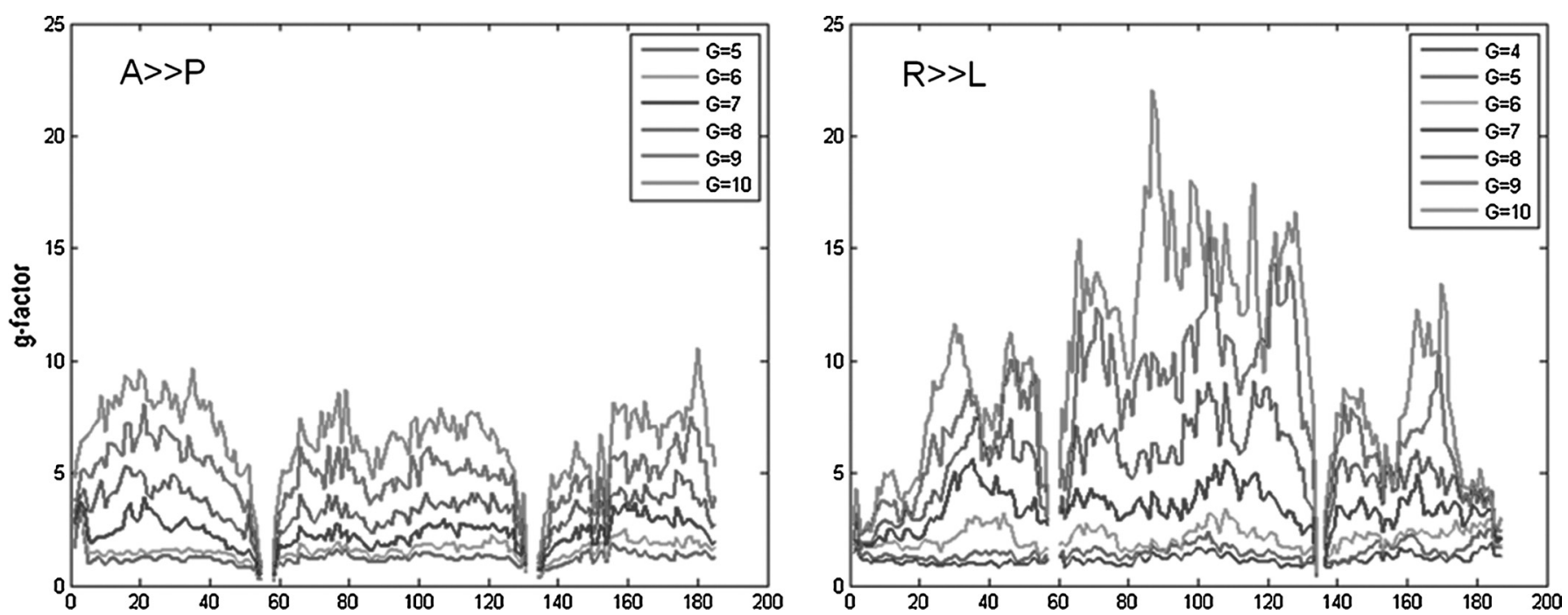

FIGURE 2. g-factor profiles for acceleration factors of $R=5$ to 10 using an internal reference scan, GRAPPA reconstruction, and $A>>P$ (left) and R $>>$ L (right) phase-encoding directions. Smaller g-factors and, hence, higher SNR are obtained from A $>>P$ encoding in this setup.

ESV, EF, and LVM and moderate for PFR and PER. In general, ICCs are lower for the low- and high-resolution protocols rtLRInt and rtHRSep.

\section{In Vivo Study: Acquisition Time}

Depending on the number of slices acquired for ventricular coverage as well as the volunteer heart rate, acquisition time for whole-heart coverage was $\sim 25$ seconds and less than 30 seconds for all RT protocols, whereas for the segmented single-slice protocol,

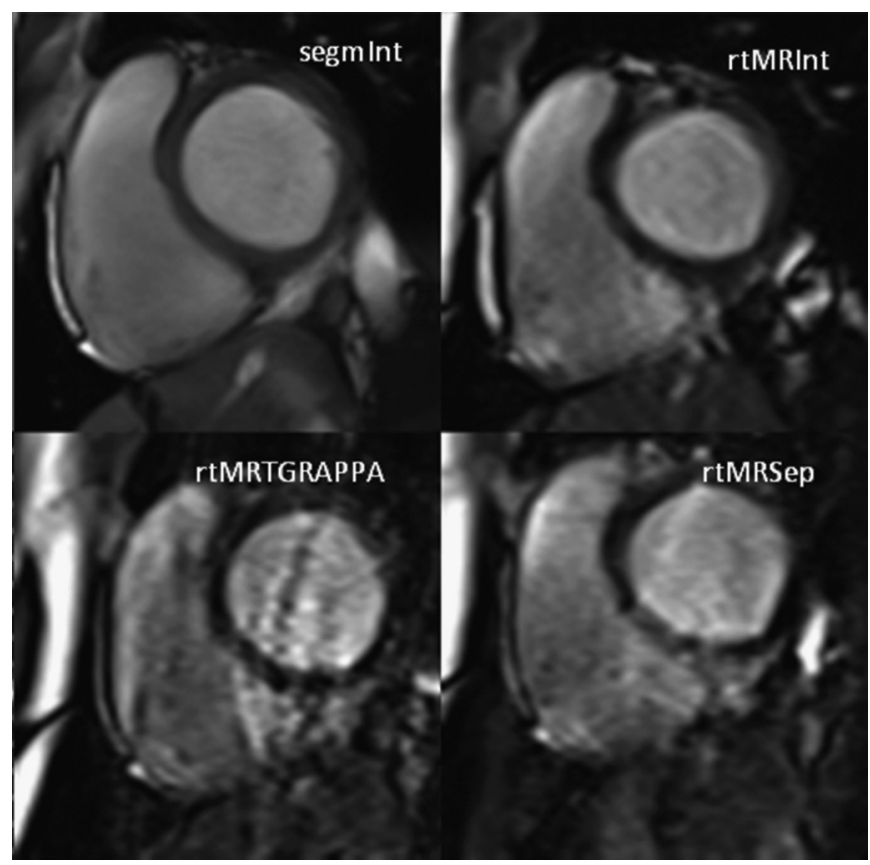

FIGURE 3. In vivo comparison of different reference scan acquisition methods using GRAPPA reconstruction: Segmented k-space readout with internal reference scan (segmlnt) and RT acquisitions with internal reference scan (rtMRInt), TGRAPPA (rtMRTGRAPPA), and separate reference scan (rtMRSep). it ranged from 5 to 8 minutes, including breath holds and recovery periods. The length of the separate reference scan was no longer than 2 seconds.

\section{Assessment of Image Quality}

Median image scores were as follows: segmInt, 1 (range, 1-1); rtLRInt, 1 (range, 1-3); rtMRSep, 1 (range, 1-4; 1 volunteer nondiagnostic, image quality rated 2 in second experiment); and rtHRSep, 2.5 (range, $2-4 ; 3$ volunteers rated nondiagnostic, all rated 3 in second experiment).

\section{DISCUSSION}

The results of this study are in accordance with and do augment the results of previous examinations $s^{4,26,27}$ by moving to $3 \mathrm{~T}$ as

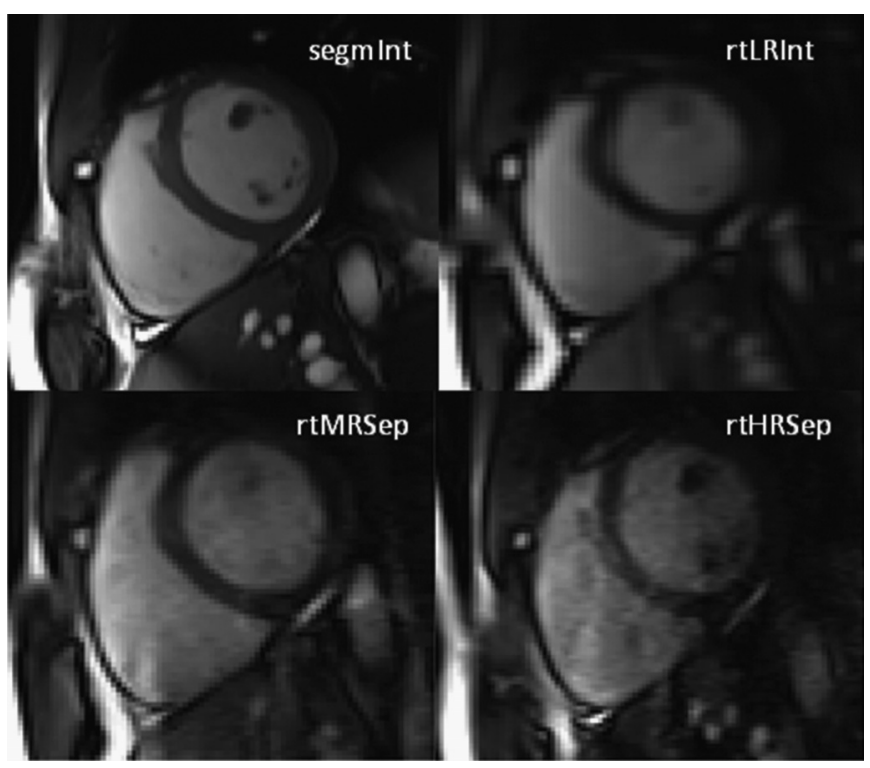

FIGURE 4. Exemplary comparison of image quality of segmented acquisition (standard of reference) and all 3 RT protocols. 
TABLE 2. Mean \pm SD Values and $P$ Values of Quantitative Cardiac Function Evaluation

\begin{tabular}{|c|c|c|c|c|c|c|c|}
\hline & $\begin{array}{c}\text { segmInt } \\
\text { (Reference) }\end{array}$ & rtLRInt & rtMRSep & rtHRSep & $\begin{array}{l}P \text { (Reference vs } \\
\text { rtLRInt) }\end{array}$ & $\begin{array}{c}P \text { (Reference vs } \\
\text { rtMRSep) }\end{array}$ & $\begin{array}{c}P \text { (Reference vs } \\
\text { rtHRSep) }\end{array}$ \\
\hline $\mathrm{EDV}, \mathrm{mL}$ & $140.7 \pm 24.1$ & $138.3 \pm 21.4$ & $137.7 \pm 19.1$ & $127.8 \pm 33.1$ & 0.7 & 0.66 & 0.36 \\
\hline $\mathrm{ESV}, \mathrm{mL}$ & $55.3 \pm 14.6$ & $60.6 \pm 14.0$ & $58.0 \pm 11.9$ & $54.5 \pm 19.7$ & 0.23 & 0.43 & 0.62 \\
\hline $\mathrm{EF}, \%$ & $61.1 \pm 4.9$ & $56.6 \pm 5.0$ & $58.2 \pm 4.4$ & $55.9 \pm 7.8$ & 0.01 & 0.11 & 0.06 \\
\hline PER, $\mathrm{mL} / \mathrm{s}$ & $481 \pm 73$ & $425 \pm 62$ & $434 \pm 67$ & $381 \pm 86$ & 0.03 & 0.04 & 0.01 \\
\hline $\mathrm{PFR}, \mathrm{mL} / \mathrm{s}$ & $555 \pm 79$ & $480 \pm 68$ & $500 \pm 70$ & $438 \pm 108$ & 0.007 & 0.047 & 0.004 \\
\hline LVM, g & $136.6 \pm 26.0$ & $140.6 \pm 24.9$ & $140.9 \pm 23.2$ & $130.2 \pm 30.9$ & 0.62 & 0.54 & 0.99 \\
\hline
\end{tabular}

well as by the additional analysis of the functional parameters PER, PFR, and LVM.

This study investigates the potential of highly accelerated RT protocols with parallel imaging for the quantitative analysis of global cardiac function and demonstrates that the results for EDV, ESV, and EF are consistent with those obtained by standard techniques. The low EF value of the protocol rtLRInt is presumably due to the low spatial resolution or the fact that the true in-plane pixels are more rectangular than in the other protocols, despite the use of zero filling to interpolate to squared pixels. In addition, the LVM tends to appear larger for lower spatial resolutions. This effect is even more pronounced when manual interaction during segmentation is reduced. On the other hand, as shown by the BlandAltman analysis as well as the low ICC values, the scattering of the results is usually largest for the protocol rtHRSep, presumably because of lower SNR values. Finally, as confirmed by the qualitative analysis, this protocol, with the highest repetition time, is also most sensitive to banding artifacts and, thus, to "outliers," that is, single points that differ rather strongly. No attempt was made to exclude these points in the interest of obtaining a representative overview of all protocols. These quantitative results, along with the assessment of general image quality, lead to the conclusion that the protocol rtMRSep is preferable to both alternatives. In comparison, the results of the protocols rtLRInt and rtHRSep also demonstrate that an increase in SNR (rtLRInt) or spatial resolution (rtHRSep) does not improve the results because, invariably, tradeoffs are involved.

An important aspect considered in detail in this work is the influence of different parallel imaging methods, including the reference scan acquisition as well as the details of the reconstruction algorithms. To obtain correct results for EDV and especially ESV, a temporal resolution of 50 milliseconds or better is necessary, ${ }^{6}$ which corresponds to about 20 images per cardiac cycle at a heart rate of 60 beats per minute. To achieve this, it is indispensable to use parallel imaging tools. The two most common methods are SENSE and GRAPPA, where image reconstruction is performed in image space and k-space, respectively. To do so, information about the coil sensitivity is required in both cases, which can be obtained by acquiring additional reference lines in the center of $\mathrm{k}$-space or, alternatively, by using temporal parallel acquisition techniques (tPATs) known as TSENSE or TGRAPPA. The reference scan can be performed either internally, that is, by acquiring reference lines for each image, or externally, in which case it is performed separately before acquiring the undersampled images. Temporal parallel acquisition technique requires no reference scan because the coil sensitivity profiles are calculated over several time points by using a "sliding window" approach: Each image is undersampled, but by combining images from several consecutive time points, in which the recorded lines are shifted, one can obtain the complete coil sensitivity information. It is important to note that the full acceleration as given by the reduction factor $R$ can be achieved only by using a separate reference scan or tPAT because the acquisition of reference lines per image increases the acquisition time for each image. For example, using a base resolution of 128 and a reduction factor of $R=4$ results in a net acceleration $R_{\text {eff }}=2.91$ if 12 reference lines are taken per image. Therefore, temporal resolution can be improved by using a separate scan or tPAT, but these approaches are more susceptible to motion artifacts because image reconstruction effectively takes place over several points in time. An alternative technique that does not involve parallel imaging but can also be used to reduce temporal resolution is view sharing. Here, only the center of k-space is acquired for every image and the outer k-space is shared between neighboring images. In this study, view sharing has been avoided whenever possible to obtain a more exact temporal resolution and avoid interpolation.

Previous studies ${ }^{12,16,28}$ have investigated the influence of these methods on the possible acceleration and the SNR properties for segmented single-slice protocols. In the context of RT protocols, dedicated studies using the combination of GRAPPA reconstruction and an internal reference scan as well as using TGRAPPA ${ }^{26}$ or TSENSE ${ }^{27}$ exist (recently, modifications KL-TSENSE and KLTGRAPPA have also been used ${ }^{29}$ ). Acquisitions with internal reference scan, although yielding the optimal image quality, necessarily limit either temporal or spatial resolution and cannot be improved much beyond the sequence protocol rtLRInt presented in this work without invoking further technical advances, such as a possibility to reduce the number of reference lines while achieving sufficient image quality. Therefore, the current study investigates alternative reconstruction methods to improve spatial resolution without sacrificing temporal resolution. In this context, the optimal method found was to acquire a separate reference scan before the actual undersampled image acquisition. Although the corresponding images are sometimes afflicted with slight motion artifacts, the quantitative results show that these are benign enough to still allow for a correct segmentation of the left ventricle.

This study also shows where limits of this method currently occur: At an acceleration factor of 6 , images are quite noisy with impaired image quality. Because the g-factors of the cardiac coil in use for this study are $\sim 1$ for these acceleration factors, further improvement could therefore be obtained only by increasing the baseline SNR. This aspect has been addressed in the present study by using a geometrically optimized 32-channel cardiac coil as well as the use of 3-T external field strength. In addition, we investigated whether RT protocols can also be used to monitor the complete VTC and determine PEF and PFR, thereby obtaining a more extensive analysis of the global LV function. These parameters cannot be assessed as reliably as the standard parameters EDV, ESV, and EF, as shown by the somewhat lower ICC values even for the best protocol rtMRSep. In addition, we observed a significant difference compared with the results from standard of reference. At the moment, it is not clear whether this results from an insufficient temporal or spatial resolution, the better image quality of the gold standard 

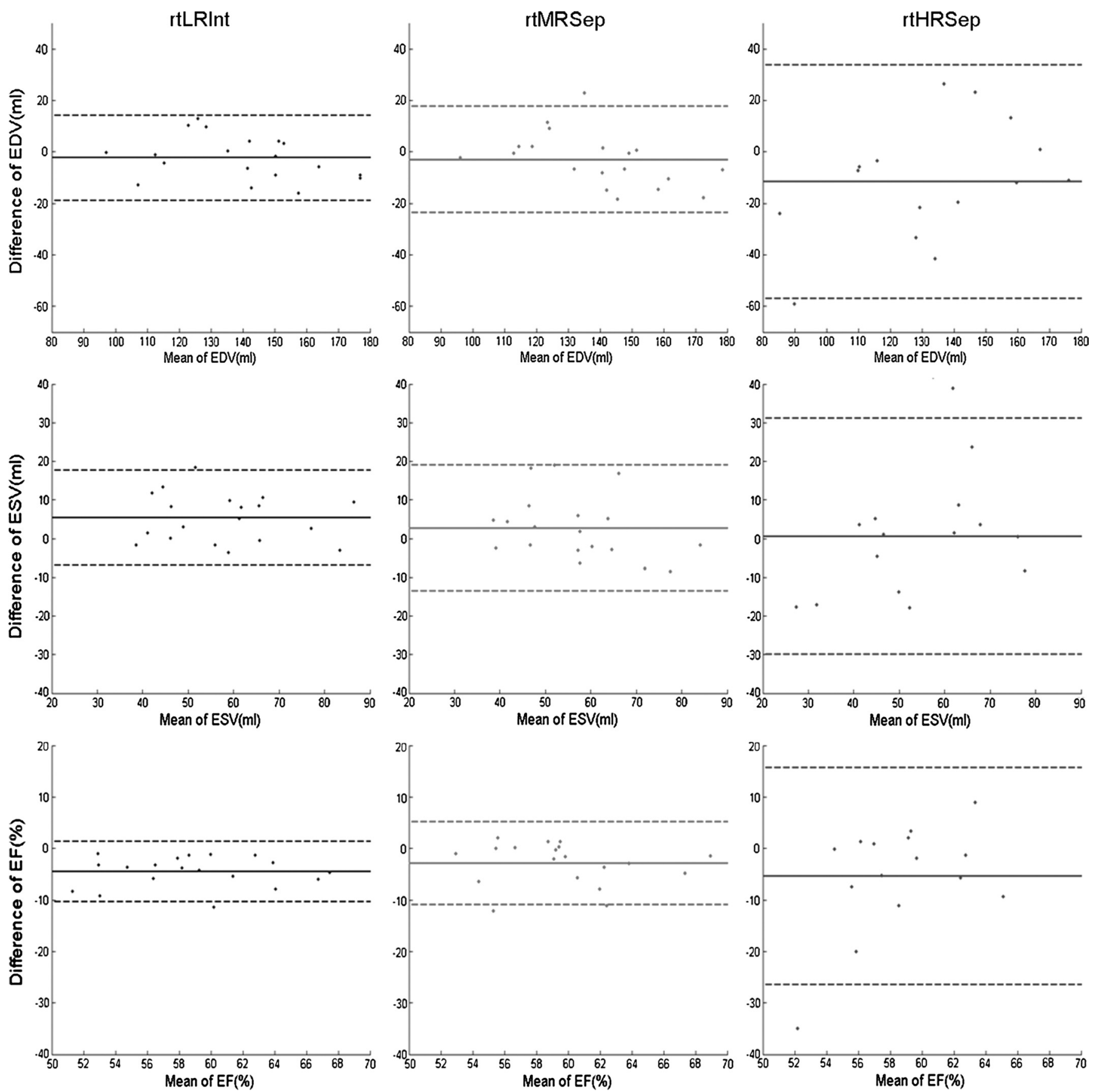

FIGURE 5. Bland-Altman plots for the values of the LV EDV, ESV, and EF for all RT protocols, comparing each with the segmented readout standard of reference. Solid lines are drawn for the mean differences, whereas dashed line corresponds to \pm 1.96 times the standard deviation.

protocol, or whether this is simply a statistical effect that is more pronounced because of the larger uncertainties in the determination of these parameters.

Previous studies of functional cardiac RT imaging have been performed mainly at $1.5 \mathrm{~T}$. Accordingly, spatial resolutions obtained were significantly lower than those presented in this work, but functional protocols at $1.5 \mathrm{~T}$ are less susceptible to banding artifacts. As an example, we quote the results given in Wintersperger et al, ${ }^{9}$ where a spatial resolution of $3.1 \times 4.2 \times 10 \mathrm{~mm}^{3}$ (corresponding to a matrix size of $128 \times 48$ ) and a temporal resolution of 48 milliseconds are obtained using GRAPPA reconstruction with an internal reference scan at an acceleration factor of $R=2$ and view sharing.

On the other hand, results similar to those obtained in this work have been presented ${ }^{27}$ using TSENSE reconstruction $(R=4)$ at $1.5 \mathrm{~T}$. The spatial resolution reported there is $1.9 \times 2.5 \times 10 \mathrm{~mm}^{3}$, 

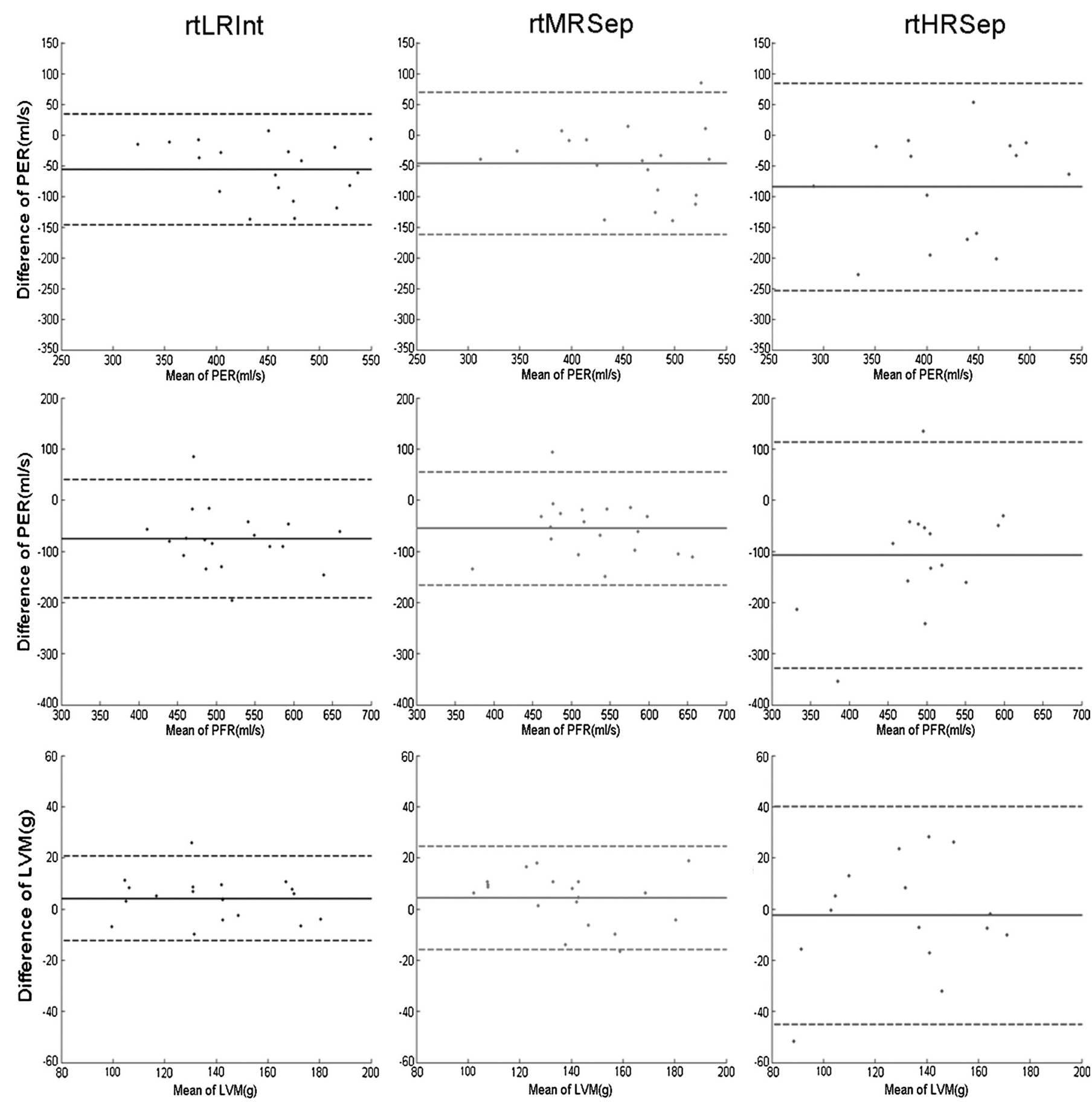

FIGURE 6. Bland-Altman plots for the values of the LV PER, PFR, and LVM for all RT protocols, comparing each with the segmented readout standard of reference. Solid lines are drawn for the mean differences, whereas dashed line corresponds to \pm 1.96 times the standard deviation.

also at a temporal resolution of 48 milliseconds. In contrast to the work presented here, that study used a dual breath-hold technique instead of free breathing. The results using TGRAPPA ${ }^{26}$ are very similar, with a spatial resolution of $1.9 \times 2.7 \times 10 \mathrm{~mm}^{3}$ reported using an acceleration factor of $R=3$ and also at $1.5 \mathrm{~T}$. There, the images are acquired using a single breath-hold. However, the acquisition time reported is $28 \pm 6$ seconds, which cardiac patients must be expected to find difficult.
There are several alternative approaches for RT cardiac MRI. Recently, the combination of radial acquisition with parallel imaging has been presented as a promising alternative. ${ }^{30}$ To calculate the GRAPPA kernels, a prescan is performed, consisting of several fully sampled images at several points in time. Then, kernels can be determined for each spatial point. In a further extension of this approach using a spiral $\mathrm{k}$-space readout, ${ }^{31}$ a temporal resolution of 29 milliseconds for spatial resolution of better than $2 \mathrm{~mm}$ is reported. 
TABLE 3. Systematic and Random Differences as Depicted in the Bland-Altman Plots for All Quantities and Protocols Investigated in This Work

\begin{tabular}{|c|c|c|c|}
\hline & Systematic (+ Random) Difference & Systematic (+ Random) Difference & Systematic (+ Random) Difference \\
\hline & rtLRInt & rtMRSep & rtHRSep \\
\hline $\mathrm{EDV}, \mathrm{mL}$ & $-2.4 \pm 8.4$ & $-3.0 \pm 10.5$ & $-11.6 \pm 23.1$ \\
\hline $\mathrm{EF}, \%$ & $-4.5 \pm 3.0$ & $-2.9 \pm 4.1$ & $-5.4 \pm 10.8$ \\
\hline $\mathrm{PER}, \mathrm{mL} / \mathrm{s}$ & $-56.7 \pm 46.0$ & $-46.9 \pm 59.2$ & $-85.1 \pm 86.1$ \\
\hline $\mathrm{PFR}, \mathrm{mL} / \mathrm{s}$ & $-75.6 \pm 59.1$ & $-55.7 \pm 56.4$ & $-108.3 \pm 112.6$ \\
\hline
\end{tabular}

Alternatively, newer reconstruction techniques can be used to obtain high temporal resolutions from radial sampling. ${ }^{32}$ However, these methods require extensive hardware for image reconstruction and are therefore not available on most installed scanners. Finally, potentially interesting advances in RT cardiac imaging have been obtained by the use of compressed sensing. ${ }^{33-35}$

None of these previous studies have attempted to analyze the parameters PER, PFR, and LVM in the context of RT measurements. These are, in particular, interesting in patients with hypertension and coronary artery disease, in whom PFR values have been shown to differ significantly from those of healthy volunteers, whereas the EF does not. ${ }^{36}$ In addition, because these parameters are more sensitive to the segmentation procedure, they also offer a stronger test of the image quality of the RT protocols.

\section{CONCLUSIONS}

With the use of a dedicated cardiac coil and by applying a separate reference scan, multislice RT measurements permit a complete functional analysis of the left ventricle in free breathing with significant reductions in acquisition time. In particular, the use of high acceleration factors of up to $R=6$ allow for high spatial and temporal resolution, and motion artifacts from the use of the separate reference scan do not impede image segmentation. This work presents protocols that provide values for the LV ESV, EDV, EF, and LVM, which are consistent with standard of reference data and are thus suitable for clinical use. Additional parameters that describe in more detail the volume time curve, such as PFR and PER, can also be obtained from the presented protocols, albeit with larger statistical uncertainties. This technique might even be used to extend functional analysis by MRI to patients with cardiac arrhythmias.

TABLE 4. ICCs for All Protocols and All Quantities, Assessing Intraobserver and Interobserver Agreement, as well as Reproducibility

\begin{tabular}{lccccccc}
\hline \multirow{5}{*}{ segmInt } & EDV & ESV & EF & PFR & PER & LVM \\
& Intraobserver & 0.96 & 0.90 & 0.77 & 0.97 & 0.88 & 0.81 \\
& Interobserver & 0.93 & 0.87 & 0.71 & 0.84 & 0.76 & 0.85 \\
rtLRInt & Reproducibility & 0.90 & 0.80 & 0.70 & 0.87 & 0.65 & 0.87 \\
& Intraobserver & 0.97 & 0.87 & 0.58 & 0.95 & 0.94 & 0.87 \\
& Interobserver & 0.91 & 0.80 & 0.62 & 0.74 & 0.73 & 0.75 \\
& Reproducibility & 0.93 & 0.82 & 0.58 & 0.51 & 0.66 & 0.78 \\
rtMRSep & Intraobserver & 0.99 & 0.95 & 0.76 & 0.84 & 0.95 & 0.87 \\
& Interobserver & 0.86 & 0.82 & 0.70 & 0.91 & 0.88 & 0.68 \\
& Reproducibility & 0.84 & 0.83 & 0.70 & 0.70 & 0.60 & 0.80 \\
rtHRSep & Intraobserver & 0.84 & 0.74 & 0.50 & 0.77 & 0.31 & 0.82 \\
& Interobserver & 0.83 & 0.80 & 0.53 & 0.86 & 0.62 & 0.64 \\
& Reproducibility & 0.83 & 0.88 & 0.21 & 0.44 & 0.40 & 0.89 \\
\hline
\end{tabular}

\section{REFERENCES}

1. Knauth AL, Gauvreau K, Powell AJ, et al. Ventricular size and function assessed by cardiac MRI predict major adverse clinical outcomes late after tetralogy of Fallot repair. Heart. 2008;94:211-216.

2. Bolli R, Chugh AR, D'Amario D, et al. Cardiac stem cells in patients with ischaemic cardiomyopathy (SCIPIO): initial results of a randomised phase 1 trial. Lancet. 2011;378:1847-1857. http://www.sciencedirect.com/science/ article/pii/S0140673611615900.

3. Pattynama PM, Lamb HJ, van der Velde EA, et al. Left ventricular measurements with cine and spin-echo MR imaging: a study of reproducibility with variance component analysis. Radiology. 1993;187:261-268.

4. Bellenger N. Comparison of left ventricular ejection fraction and volumes in heart failure by echocardiography, radionuclide ventriculography and cardiovascular magnetic resonance. Are they interchangeable? Eur Heart J. 2000;21:1387-1396.

5. Ichikawa Y, Sakuma H, Kitagawa K, et al. Evaluation of left ventricular volumes and ejection fraction using fast steady-state cine MR imaging: comparison with left ventricular angiography. J Cardiovasc Magn Reson. 2003; 5:333-342.

6. Miller S, Simonetti O, Carr J, et al. MR Imaging of the heart with cine true fast imaging with steady-state precession: influence of spatial and temporal resolutions on left ventricular functional parameters. Radiology. 2002;223: 263-269.

7. Barkhausen J, Goyen M, Ruhm S, et al. Assessment of ventricular function with single breath-hold real-time steady-state free precession cine MR imaging. AJR Am J Roentgenol. 2002;178:731-735.

8. Lee V, Resnick D, Bundy J, et al. Cardiac function: MR evaluation in one breath hold with real-time true fast imaging with steady-state precession. Radiology. 2002;222:835-842.

9. Wintersperger B, Nikolaou K, Dietrich O, et al. Single breath-hold real-time cine MR imaging: improved temporal resolution using generalized autocalibrating partially parallel acquisition (GRAPPA) algorithm. Eur Radiol. 2003;13:1931-1936.

10. Davarpanah AH, Chen Y, Kino A, et al. Accelerated two- and three-dimensional cine MR imaging of the heart by using a 32-channel coil. Radiology. 2009;254: 98-108.

11. Sievers B, Schrader S, Hunold P, et al. Free breathing 2D multi-slice real-time gradient-echo cardiovascular magnetic resonance imaging: impact on left ventricular function measurements compared with standard multi-breath hold 2D steady-state free precession imaging. Acta Cardiol. 2011;66:489-497.

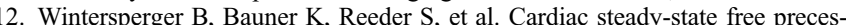
sion CINE magnetic resonance imaging at 3.0 Tesla: impact of parallel imaging acceleration on volumetric accuracy and signal parameters. Invest Radiol. 2006; $41: 141-147$

13. Schar M, Kozerke S, Fischer S, et al. Cardiac SSFP imaging at 3 Tesla. Magn Reson Med. 2004;51:799-806.

14. Fenchel M, Deshpande V, Nael K, et al. Cardiac cine imaging at 3 Tesla: initial experience with a 32-element body-array coil. Invest Radiol. 2006;41: 601-608.

15. Sandner T, Houck P, Runge V, et al. Accuracy of accelerated cine MR imaging at 3 Tesla in longitudinal follow-up of cardiac function. Eur Radiol. 2008; 18:2095-2101

16. Wintersperger B, Reeder S, Nikolaou K, et al. Cardiac CINE MR imaging with a 32-channel cardiac coil and parallel imaging: impact of acceleration factors on image quality and volumetric accuracy. J Magn Reson Imaging. 2006; 23:222-227.

17. Michaely H, Nael K, Schoenberg S, et al. Analysis of cardiac functioncomparison between 1.5 Tesla and 3.0 Tesla cardiac cine magnetic resonance imaging: preliminary experience. Invest Radiol. 2006;41:133-140.

18. Tsutsui H, Tsuchihashi M, Takeshita A. Mortality and readmission of hospitalized patients with congestive heart failure and preserved versus depressed systolic function. Am J Cardiol. 2001;88:530-533. 
19. Griswold M, Jakob P, Heidemann R, et al. Generalized autocalibrating partially parallel acquisitions (GRAPPA). Magn Reson Med. 2002;47:1202-1210.

20. Wang J, Kluge T, Nittka M, et al. Parallel Imaging Techniques with modified SENSE reconstruction mSENSE. In: Proceedings of the first Würzburg Workshop on Parallel Imaging Basics and Clinical Applications, Würzburg. 2001:Abstract 89.

21. Breuer FA, Kellman P, Griswold MA, et al. Dynamic autocalibrated parallel imaging using temporal GRAPPA (TGRAPPA). Magn Reson Med. 2005;53:981-985.

22. Pruessmann KP, Weiger M, Scheidegger MB, et al. SENSE: sensitivity encoding for fast MRI. Magn Reson Med. 1999;42:952-962.

23. Breuer F, Kannengiesser S, Blaimer M, et al. General formulation for quantitative G-factor calculation in GRAPPA reconstructions. Magn Reson Med. 2009;62:739-746.

24. Reeder S, Wintersperger B, Dietrich O, et al. Practical approaches to the evaluation of signal-to-noise ratio performance with parallel imaging: application with cardiac imaging and a 32-channel cardiac coil. Magn Reson Med. 2005;54:748-754.

25. Heiberg E, Sjogren J, Ugander M, et al. Design and validation of segmentfreely available software for cardiovascular image analysis. BMC Med Imaging. 2010;10:1.

26. Nassenstein K, Eberle H, Maderwald S, et al. Single breath-hold magnetic resonance cine imaging for fast assessment of global and regional left ventricular function in clinical routine. Eur Radiol. 2010;20:2341-2347.

27. Wintersperger B, Sincleair S, Runge V, et al. Dual breath-hold magnetic resonance cine evaluation of global and regional cardiac function. Eur Radiol. $2007 ; 17: 73-80$
28. Hunold P, Maderwald S, Ladd M, et al. Parallel acquisition techniques in cardiac cine magnetic resonance imaging using TrueFISP sequences: comparison of image quality and artifacts. J Magn Reson Imaging. 2004;20: 506-511.

29. Ding Y, Chung Y, Jekic M, et al. A new approach to autocalibrated dynamic parallel imaging based on the Karhunen-Loeve transform: KL-TSENSE and KL-TGRAPPA. Magn Reson Med. 2011;65:1786-1792.

30. Seiberlich N, Ehses P, Duerk J, et al. Improved radial GRAPPA calibration for real-time free-breathing cardiac imaging. Magn Reson Med. 2011;65:492-505.

31. Seiberlich N, Lee G, Ehses P, et al. Improved temporal resolution in cardiac imaging using through-time spiral GRAPPA. Magn Reson Med. 2011;66: $1682-1688$.

32. Zhang S, Uecker M, Voit D, et al. Real-time cardiovascular magnetic resonance at high temporal resolution: radial FLASH with nonlinear inverse reconstruction. J Cardiovasc Magn Reson. 2010;12:39.

33. Wech T, Gutberlet M, Greiser A, et al. Hochaufgeloste funktionelle Herz-MR Bildgebung mithilfe dichtegewichteter Echtzeit-Datenaufnahme und einer Kombination von Compressed Sensing und paralleler Bildgebung in der Rekonstruktion. Rofo. 2010;182:676-681.

34. Wech T, Lemke A, Medway D, et al. Accelerating cine-MR imaging in mouse hearts using compressed sensing. J Magn Reson Imaging. 2011;34: 1072-1079.

35. Usman M, Atkinson D, Odille F, et al. Motion corrected compressed sensing for free-breathing dynamic cardiac MRI. Magn Reson Med. 2012. [Epub ahead of print].

36. Zeidan Z, Erbel R, Barkhausen J, et al. Analysis of global systolic and diastolic left ventricular performance using volume-time curves by real-time threedimensional echocardiography. J Am Soc Echocardiogr. 2003;16:29-37. 